\title{
Habilidades pragmáticas em crianças deficientes auditivas: estudo de casos e controles
}

\author{
Pragmatic abilities in hearing impaired children: a case- \\ control study
}

\author{
Luana Curti' ${ }^{1}$, Taiana d'Ávila Quintas ${ }^{2}$, Bárbara Niegia Garcia Goulart ${ }^{3}$, Brasilia Maria Chiari ${ }^{4}$
}

\begin{abstract}
RESUMO
Objetivo: Avaliar as habilidades pragmáticas de um grupo de crianças deficientes auditivas e compará-las a seus pares normoouvintes. Métodos: Estudo de casos e controles composto por 32 crianças de ambos os gêneros com idades entre dois e seis anos. Dentre estas, 16 deficientes auditivas de grau moderadamente severo a profundo sem outros comprometimentos orgânicos (casos) e 16 crianças normo-ouvintes sem queixas fonoaudiológicas (controle) pareadas por idade. A avaliação e a análise da pragmática foram realizadas a partir do Teste ABFW-Pragmática, seguindo as instruções do protocolo. Resultados: A média de idade das crianças estudadas foi quatro anos ( $\mathrm{DP}=1,3)$; houve diferença significativa em relação ao número de atos comunicativos por minuto entre casos e controles $(\mathrm{p}=0,001)$. As crianças deficientes auditivas apresentaram menos iniciativas comunicativas do que as crianças normo-ouvintes e o meio comunicativo gestual foi utilizado por $13(81,3 \%)$ destas e por cinco $(32,2 \%)$ das crianças normo-ouvintes $(\mathrm{p}=0,004)$. Não houve diferença entre os grupos em relação às intenções comunicativas $(\mathrm{p}=0,465)$. Conclusão: As crianças deficientes auditivas foram capazes de interagir em situações contextualizadas utilizando-se de funções comunicativas semelhantes às das ouvintes, e se diferiram destas quanto ao meio comunicativo mais utilizado.
\end{abstract}

Descritores: Surdez; Desenvolvimento de linguagem; Linguagem; Avaliação; Comunicação não verbal; Deficiência auditiva; Desenvolvimento infantil; Testes de linguagem

\section{INTRODUÇÃO}

A audição é o que nos torna comunicadores eficazes por meio do tipo mais comum da linguagem, a verbal. A falta deste sentido não nos impede de organizar a realidade experimentada, mas acarreta grandes dificuldades que não se limitam apenas à alteração auditiva, mas se expandem a desvios nos desenvolvimentos social, psíquico e educacional.

Para que a aquisição e o desenvolvimento da linguagem

Trabalho realizado no Curso de Fonoaudiologia da Universidade Federal de São Paulo - UNIFESP - São Paulo (SP), Brasil, com bolsa concedida pela Fundação de Amparo à Pesquisa do Estado de São Paulo (FAPESP).

(1) Pós-graduanda (Especialização) em Voz pelo Centro de Estudos da Voz - CEV - São Paulo (SP), Brasil.

(2) Fonoaudióloga formada pela Universidade Federal de São Paulo UNIFESP - São Paulo (SP), Brasil.

(3) Doutora, Professora Adjunto da Universidade Federal do Rio Grande do Sul - UFRGS - Porto Alegre (RS), Brasil; Pesquisadora Colaboradora do Departamento de Fonoaudiologia da Universidade Federal de São Paulo UNIFESP - São Paulo (SP), Brasil.

(4) Livre-docente, Professora Titular do Departamento de Fonoaudiologia da Universidade Federal de São Paulo - UNIFESP - São Paulo (SP), Brasil. Endereço para correspondência: Luana Curti. R. Cachoeira do Sul, 271/102A, Vila Jaguara, São Paulo (SP), Brasil, CEP: 05117-010. E-mail: luana.curti@hotmail.com

Recebido em: 07/05/2009; Aceito em: 19/11/2009 sejam normais, é pré-requisito que o sistema auditivo esteja íntegro anátomo e fisiologicamente. $\mathrm{O}$ início precoce de uma perda auditiva pode ter consequências devastadoras no desenvolvimento da competência comunicativa ${ }^{(1,2)}$ como diversos problemas de fala, relacionados tanto à emissão quanto à compreensão, e de linguagem, que são observados nos deficientes auditivos.

Ao determinar o impacto da deficiência auditiva na vida de uma pessoa, é fundamental que se considere o efeito da perda auditiva na habilidade de se comunicar ${ }^{(1)}$.

A pragmática é uma habilidade comunicativa e social. É o aspecto funcional da comunicação ${ }^{(3,4)}$.

Para que a criança adquira linguagem é necessário que aprenda estruturas abstratas da competência linguística e que as use apropriadamente ${ }^{(5,6)}$.

A perspectiva pragmática contribui para a inserção da comunicação não-verbal nos estudos da linguagem infantil, considerando que é no período pré-linguístico que se inicia o uso da linguagem para interação social e que se estabelecem as bases funcionais da comunicação ${ }^{(4)}$.

O profissional de Fonoaudiologia deve avaliar a relação entre a habilidade de linguagem e a competência comunicativa. A habilidade de linguagem refere-se à capacidade da criança em reconhecer e formular os sistemas simbólicos, tanto falados 
como escritos, já a competência comunicativa compreende a habilidade de fazer o uso da linguagem com intenção de interação entre contextos sociais ${ }^{(7)}$.

Utilizar-se da análise pragmática permite ao fonoaudiólogo a determinação de como e quando o indivíduo utiliza suas funções comunicativas ${ }^{(3,8)}$, podendo assim, definir se uma criança é, ou pode vir a ser, um comunicador eficaz, obtendo melhor diagnóstico e controle evolutivo das crianças deficientes auditivas ${ }^{(9)}$.

Este estudo pretende avaliar as habilidades pragmáticas de um grupo de crianças deficientes auditivas protetizadas e compará-las com seus pares normo-ouvintes.

\section{MÉTODOS}

Esta pesquisa foi aprovada pelo Comitê de Ética em Pesquisa (CEP) da Universidade Federal de São Paulo sob o protocolo número $n^{\circ} 1363 / 07$. Os responsáveis pelas crianças participantes do estudo assinaram Termo de Consentimento Livre e Esclarecido e todas as normas da resolução 196/96 do Conselho Nacional de Saúde foram respeitadas pelos pesquisadores responsáveis pelo estudo.

Os casos foram localizados a partir de contato com os responsáveis pelas crianças elegíveis para o estudo e que frequentavam o Laboratório de Investigação da Linguagem nos Distúrbios da Audição do Departamento de Fonoaudiologia da Universidade Federal de São Paulo e, para o grupo controle, crianças de escolas regulares do estado de São Paulo na época da coleta de dados.

Desta forma, este é um estudo de casos e controles composto por 32 crianças de ambos os sexos com idades entre dois e seis anos. Dentre estas, 16 deficientes auditivas de grau moderadamente severo a profundo sem outros comprometimentos (casos) e 16 crianças normo-ouvintes sem queixas fonoaudiológicas ou histórico de fonoterapia (controle) pareadas por idade.

As habilidades pragmáticas foram avaliadas pelo Teste de Linguagem Infantil - ABFW - Pragmática ${ }^{(10)}$. A coleta dos dados foi feita por meio de gravação em vídeo de um segmento de 30 minutos de interação com um adulto familiar à criança, em situação livre com brinquedos disponíveis, como bonecas, carrinhos, bolas, peças de encaixe e alguns objetos quebrados, como forma de estímulo para o início da interação, conforme orientações do manual do teste utilizado. O grupo controle teve a filmagem realizada na própria escola da criança em situação de interação com um adulto familiar (com o professor ou o cuidador). Os deficientes auditivos (grupo de casos) tiveram como cenário da filmagem a sala de fonoterapia, ambiente com o qual já estavam familiarizados em virtude de estar em acompanhamento fonoaudiológico, utilizando o mesmo material disponibilizado para o grupo controle (ouvintes), em situação de interação com o cuidador ou com o fonoterapeuta. A gravação foi realizada com uma filmadora da marca Panasonic $\AA$, modelo Palmcorder - IQ com fitas cassetes compactas da marca JVC®. Foram analisados atos, meios e funções comunicativas realizadas pelas crianças durante a gravação.

Foram considerados atos comunicativos quando houve interação adulto-criança, criança-adulto ou criança-objeto até o seu término. Os meios comunicativos poderiam ser verbal (envolvendo pelo menos $75 \%$ de fonemas), vocal (qualquer outra emissão) ou ainda, gestual (por movimentos do rosto ou do corpo $)^{(10)}$. As funções comunicativas refletem a competência funcional da interação, pois são caracterizadas pelo tipo de função social que os atos comunicativos representam.

Com todos os dados devidamente registrados, estes foram analisados qualitativamente e quantitativamente, por meio de tratamento estatístico, com os Softwares SPSS versão 11.5 e EpiInfo versão 3.5.1, comparando as habilidades pragmáticas entre o grupo de casos e grupo controle.

\section{RESULTADOS}

Foram analisadas 32 crianças, com idade média de 3,92 $(\mathrm{DP}=1,35)$ no grupo de casos (deficientes auditivos) e 4,03 $(\mathrm{DP}=1,32)$ no grupo controle.

Todos os deficientes auditivos utilizavam prótese auditiva retro-auricular há, pelo menos, seis meses. Neste grupo, as crianças com quatro anos ou mais (50\% do grupo) estavam em terapia fonoaudiológica em período superior a um ano; aquelas com idade entre dois e quatro anos estavam ingressando no processo de fonoterapia. Em ambas as situações a estimulação da linguagem tinha o foco na comunicação verbal sem a utilização da LIBRAS (Língua Brasileira de Sinais).

Foi analisado o número de atos comunicativos por minuto e comparados os desempenhos entre as amostras.

Tabela 1. Comparação do grupo pesquisa e grupo controle quanto ao número de atos comunicativos por minuto

\begin{tabular}{lccc}
\hline $\begin{array}{l}\text { Número de atos } \\
\text { comunicativos/ } \\
\text { minuto }\end{array}$ & $\begin{array}{c}\text { Deficientes } \\
\text { auditivos (casos) }\end{array}$ & $\begin{array}{c}\text { Normo-ouvintes } \\
\text { (controles) }\end{array}$ & Valor de p \\
\hline Média & 2,23 & 3,34 & $<0,001^{*}$ \\
Mediana & 2,25 & 3,4 & \\
DP & 0,65 & 0,66 & - \\
\hline Total & 16 & 16 & - \\
\hline
\end{tabular}

Teste Anova

* Valor significativo $(p \leq 0,05)$

Legenda: $\mathrm{DP}=$ desvio-padrão

Pode-se verificar que existe diferença estatística entre os grupos para a média de número de atos comunicativos por minuto, na qual o grupo controle possui um resultado maior do que o pesquisa, ou seja, $3,34 \pm 0,32$ versus $2,23(\mathrm{DP}=0,32)$ (Tabela 1). Vale ressaltar que apenas uma criança $(6,25 \%)$ do grupo pesquisa obteve resultado dentro do esperado para a idade e nenhuma das crianças do grupo controle atingiu o número de atos comunicativos por minuto descrito no protocolo ABFW-Pragmática.

Em seguida, foi realizada uma comparação entre os grupos para os resultados qualitativos, onde foi feito o uso do teste de Igualdade de Duas Proporções. A Tabela 2 mostra as funções comunicativas utilizadas predominantemente pelos grupos.

As funções comunicativas foram classificadas como mais ou menos interacionais, sendo Comentário, Exibição e Pedido de Ação mais interacionais, e Exploratório e Performativo menos interacionais (Tabela 3). 
Tabela 2. Comparação do grupo pesquisa e grupo controle quanto a função comunicativa predominante

\begin{tabular}{lccccc}
\hline Função comunicativa & \multicolumn{2}{c}{ Deficientes auditivos (Casos) } & \multicolumn{2}{c}{ Normo-ouvintes (Controles) } & \multirow{2}{*}{ Valor de $\mathrm{p}$} \\
\cline { 2 - 5 } & Quantidade & $\%$ & Quantidade & 43,8 & $0,049^{*}$ \\
\hline Comentário & 2 & 12,5 & 7 & 0 & 0,310 \\
Exibição & 1 & 6,3 & 0 & 50 & 0,723 \\
Exploratória & 9 & 56,3 & 0 & 0 & 0,144 \\
Pedido de ação & 2 & 12,5 & 1 & 6,3 & 0,544 \\
Performativo & 2 & 12,5 & 0 & & 0 \\
\hline
\end{tabular}

Teste de Igualdade de Duas Proporções

* Valor significativo $(p \leq 0,05)$

Tabela 3. Comparação do grupo pesquisa e grupo controle quanto ao tipo de função comunicativa predominante

\begin{tabular}{lccccc}
\hline Função comunicativa predominante & \multicolumn{2}{c}{ Deficientes auditivos (casos) } & \multicolumn{2}{c}{ Normo-ouvintes (controles) } & Valor de $\mathrm{p}$ \\
\cline { 2 - 5 } & Quantidade & $\%$ & Quantidade & $\%$ & $0,465^{*}$ \\
\hline Mais interacionais & 5 & 31,30 & 7 & 43,80 & 56,30 \\
Menos interacionais & 11 & 68,80 & 9 & \\
\hline
\end{tabular}

Teste de Igualdade de Duas Proporções

*Valor significativo $(p \leq 0,05)$

Tabela 4. Comparação do grupo pesquisa e grupo controle quanto ao meio comunicativo utilizado

\begin{tabular}{lccccc}
\hline Meio predominante & \multicolumn{2}{c}{ Deficientes auditivos (casos) } & \multicolumn{2}{c}{ Normo-ouvintes (controles) } & \multirow{2}{*}{ Valor de $p$} \\
\cline { 2 - 4 } & Quantidade & $\%$ & Quantidade & $\%$ & 31,3 \\
Gestual & 13 & 81,3 & 5 & 11 & $0,004^{*}$ \\
Verbal & 3 & 18,8 & 68,8 & \\
\hline
\end{tabular}

Teste de Igualdade de Duas Proporções

* Valor significativo $(p \leq 0,05)$

Pode-se verificar que embora exista diferença entre as distribuições dos dois grupos, as mesmas não podem ser consideradas significativas para o tipo de função comunicativa utilizada.

Para finalizar, foi realizada uma comparação inter-grupo para o meio comunicativo predominante (Tabela 4).

Pode-se verificar que existe diferença entre os grupos para os meios predominantes, onde o grupo pesquisa tem maior percentual de Gestual e o grupo controle maior percentual de Verbal.

\section{DISCUSSÃO}

A avaliação clínica é uma das etapas do processo que envolve a atuação fonoaudiológica, principalmente a atuação ligada à prevenção e à reabilitação. Pressupõe-se o conhecimento e o uso de uma série de ferramentas técnicas, especificamente ligadas à Fonoaudiologia e à comunicação humana, além de questões mais abrangentes relativas ao ser humano e suas relações com o meio ${ }^{(11)}$.

A análise das habilidades pragmáticas possibilitou, neste estudo, justamente uma avaliação da forma com que as crianças deficientes auditivas se expressam e se relacionam com o meio que estão inseridas.

Os achados deste estudo demonstram que as crianças deficientes auditivas utilizaram os mesmos tipos de funções comunicativas que seus pares do grupo controle no Teste de
Linguagem Infantil ABFW-Pragmática. Entretanto, foram encontradas diferenças em relação à quantidade de atos comunicativos por minuto e o meio comunicativo utilizado. Tais achados são descritos em estudos realizados com crianças deficientes auditivas, nos quais se pôde observar que, quando comparadas a crianças normo-ouvintes, expressam-se pelos mesmos tipos de funções comunicativas, porém, com padrões de frequências diferentes. A quantidade menor de atos comunicativos deve-se a restrições de estímulos no ambiente, já que as crianças deficientes auditivas estão privadas dos inputs auditivos, diminuindo o número de estímulos e oportunidades de interagir com os interlocutores ${ }^{(3)}$.

Vale ressaltar que as funções comunicativas, em sua grande maioria, podem ser expressas por meio de gestos. Assim, as crianças deficientes auditivas têm a possibilidade de interagir eficientemente dentro do contexto através de gestos. O que foi verificado nesta pesquisa, na qual, 13 sujeitos $(81,3 \%)$ do grupo pesquisa utilizaram o meio gestual para se expressar, porém, não se limitando às funções comunicativas menos interacionais e, sim, interagindo com o interlocutor em forma de exibição, pedido de ação e comentário. Embora não tenha sido realizada análise estatística para comparação intra-grupo, as três $(18,8 \%)$ crianças deficientes auditivas que utilizaram funções comunicativas mais interacionais, bem como o meio comunicativo verbal, estavam em terapia fonoaudiológica em período superior a três anos e tempo equivalente de uso de prótese auditiva, diferindo-se do restante do grupo, que tinha 
menos de um ano de tempo médio de fonoterapia e uso de aparelho de amplificação sonora individual (AASI).

Foi verificada a existência de outros estudos que envolviam instrumentos de avaliação da pragmática, porém em grupos de crianças com diferentes distúrbios de comunicação, que não a deficiência auditiva. Na sequência, serão apresentados alguns comentários fazendo relações possíveis destes estudos com a presente pesquisa.

Estudo realizado com crianças com alteração no desenvolvimento da linguagem encontrou que estas utilizaram predominantemente o meio comunicativo verbal para se comunicar $^{(12)}$, o que difere dos deficientes auditivos desta pesquisa, dos quais, $13(81,3 \%)$ utilizaram-se de meio gestual. Isto por conta da raiz da alteração no desenvolvimento da linguagem que, sendo ela a deficiência auditiva, priva-os do estímulo auditivo e, portanto, da comunicação verbal. No que diz respeito a crianças com diagnóstico de alterações específicas de desenvolvimento da linguagem, o parâmetro em que foi observada maior evolução após terapia fonoaudiológica foi o meio comunicativo, que evidenciou ampliação no uso de verbalizações ${ }^{(13)}$. $\mathrm{O}$ mesmo resultado se espera para a terapia fonoaudiológica dos deficientes auditivos.

Em relação às diferenças qualitativas pudemos concluir que não houve discrepância entre os dois grupos, já que não existiu diferença significativa entre os tipos de funções comunicativas utilizadas pelas crianças deficientes auditivas quando comparadas às normo-ouvintes. Quando analisadas separadamente, as funções comunicativas utilizadas pelos grupos foram distintas, como a função Comentário utilizada principalmente pelo grupo controle e as funções Pedido de Ação ou Exibição utilizadas apenas pelos deficientes auditivos. No entanto, quando as funções foram classificadas em mais ou menos interativas, os grupos se equipararam.

Em estudo anterior que analisou as habilidades pragmáticas de crianças sem alterações de linguagem, foi verificado que o meio comunicativo predominante foi verbal ${ }^{(4)}$. O mesmo resultado foi obtido com as crianças normo-ouvintes desta pesquisa, das quais $11(68,8 \%)$ utilizaram o meio verbal predominantemente para se comunicar.

Foi verificada, através de estudos com o instrumento ABFW-Pragmática, a importância da avaliação da pragmática como parâmetro objetivo para considerar a evolução no processo terapêutico ${ }^{(12)}$. O mesmo pode ser pensado nos casos de crianças deficientes auditivas em processo terapêutico. Novos estudos podem ser realizados, de forma longitudinal, para a verificação da evolução terapêutica de deficientes auditivos.

Como mostram os estudos supra-citados, com grupos de crianças de risco para o desenvolvimento da linguagem, podese dizer que o teste utilizado auxilia no adequado diagnóstico fonoaudiológico, definindo se a criança em questão é ou pode vir a ser um comunicador eficaz, contribuindo, assim, para um planejamento terapêutico mais efetivo ${ }^{(9)}$.

A análise pragmática permite que o fonoaudiólogo avalie o indivíduo de forma complexa e abrangente, não se limitando a esfera biológica da comunicação humana, já que esta constitui um sistema complexo que envolve não apenas a comunicação verbal e a escrita, mas também a expressão corporal ${ }^{(14)}$.

\section{CONCLUSÃO}

Após a análise dos resultados obtidos no Teste de Linguagem Infantil ABFW-Pragmática foi verificado que as crianças deficientes auditivas pesquisadas, expressam-se e compreendem as situações de interação familiar, utilizando-se das funções comunicativas, à semelhança das normo-ouvintes. Por outro lado, diferenciam-se destas quanto ao meio comunicativo utilizado, visto que o gesto é priorizado pelo deficiente auditivo e a comunicação verbal é o meio mais utilizado pelos seus pares normo-ouvintes.

Novos estudos podem ser realizados, de forma longitudinal, para a verificação da evolução terapêutica de deficientes auditivos.

\begin{abstract}
Purpose: To evaluate the pragmatic abilities of a group of hearing impaired children, and compare them with normal-listener peers. Methods: Case-control study composed by 32 children of both genders with ages between two and six years, paired by age: 16 hearing impaired with moderately severe to profound hearing loss without other organic dysfunctions (cases), and 16 children with normal hearing with no Speech-Language Pathology complaints (control). The evaluation and analysis of pragmatic abilities were carried out based on the ABFW-Pragmatics Test, following instructions of its own protocol. Results: The average age of the studied children was four years $(\mathrm{SD}=1,3)$; there was a significant difference between cases and controls regarding the number of communicative acts per minute $(\mathrm{p}=0,001)$. The hearing impaired children presented fewer communicative initiatives than normal listeners, and the gestural communicative mean was used by $13(81.3 \%)$ of the first group and by five $(32.2 \%)$ of the latter ( $\mathrm{p}=0,004)$. There was no difference between the groups regarding communicative intentions $(\mathrm{p}=0,465)$. Conclusion: The hearing impaired children were able to interact in contextualized situations using communicative functions similar to the listeners, and differed from them in the most used communicative mean.
\end{abstract}

Keywords: Deafness; Language development; Language; Assessment; Non-verbal communication; Hearing loss; Child development; Language tests 


\section{REFERÊNCIAS}

1. Jamieson JR. O impacto da deficiência auditiva. In: Katz J, editor. Tratado de fonoaudiologia clínica. 4a ed. São Paulo: Manole; 1999. Cap. 39.

2. Costa MCM, Chiari BM. Verificação do dsempenho de crianças deficientes auditivas oralizadas em teste de vocabulário. Pró-Fono. 2006;18(2):189-96.

3. Chiari BM, Bragatto EL, Barbosa T, Strobilius R, Soares TCB. Avaliação da intencionalidade da comunicação em crianças deficientes auditivas e ouvintes entre 24 e 60 Meses. Pró-Fono. 2002;14(2):187-98.

4. Hage SRV, Resegue MM, Viveiros DCS, Pacheco EF. Análise do perfil das habilidades pragmáticas em crianças pequenas normais. Pró-Fono. 2007;19(1):49-58.

5. Dore J. A pragmatic description of early language development. J Psycholinguist Res. 1974;3(4):343-50.

6. Cervone LM, Fernandes FDM. Análise do perfil comunicativo de crianças de 4 a 5 anos na interação com o adulto. Rev Soc Bras Fonoaudiol. 2005;10(2):97-105.

7. Cardoso C, Fernandes FDM. Relação entre os aspectos sócio cognitivos e perfil funcional da comunicação em um grupo de adolescentes do espectro autístico. Pró-Fono. 2006;18(1):89-98.

8. Porto E, Limongi SCO, Santos IG, Fernandes FDM. Amostra de filmagem e análise da pragmática na síndrome de Down. Pró-Fono. 2007;19(2):159-66.
9. Goulart BNG, Chiari BM. Testes de rastreamento $\mathrm{x}$ testes de diagnóstico: atualidades no contexto da atuação fonoaudiológica. PróFono. 2007;19(2):223-32.

10. Fernandes FDM. Pragmática. In: Andrade CRF, Befi-Lopes DM, Fernandes FDM, Wertzner HF. ABFW: teste de linguagem infantil nas áreas de fonologia, vocabulário, fluência e pragmática. Carapicuíba: Pró-Fono; 2000. p.77-84.

11. Goulart BNG, Chiari BM. Avaliação clínica fonoaudiológica, integralidade e humanização: perspectivas gerais e contribuições para reflexão [revisão]. Rev Soc Bras Fonoaudiol. 2007;12(4):335-40.

12. Befi-Lopes DM, Cattoni DM, Almeida RC. Avaliação de aspectos da pragmática em crianças com alteração no desenvolvimento da linguagem. Pró-Fono. 2000;12(2):39-47.

13. Befi-Lopes DM, Puglisi ML, Rodrigues A, Giusti E, Gândara JP, Araújo K. Perfil comunicativo de crianças com alterações especificas no desenvolvimento da linguagem: caracterização longitudinal das habilidades pragmáticas. Rev Soc Bras Fonoaudiol. 2007;12(4):265-73.

14. Goulart BNG, Chiari BM. Prevalência de desordens de fala em escolares e fatores associados. Rev Saúde Pública $=$ J Public Health. 2007;41(5):726-31. 